\title{
Diagnóstico inicial da presença de metais pesados em sedimento e em organismos bentônicos da ilha de Itacolomi, PR
}

\author{
Assessment of heavy metals in sediment and benthic organisms island Itacolomi, PR
}

\author{
Renê Trombini dos Santos ${ }^{1}$ \\ Fernando da Silva Carvalho Neto ${ }^{2}$ \\ Edinalva Oliveira ${ }^{3}$ \\ Marco Aurélio da Silva Carvalho Filho ${ }^{4}$ \\ Eliane Carvalho de Vasconcelos ${ }^{5}(*)$
}

\section{Resumo}

O presente trabalho teve como objetivo fazer um diagnóstico inicial da presença de cádmio $(\mathrm{Cd})$, aresênio $(\mathrm{As})$, mercúrio $(\mathrm{Hg})$ e chumbo $(\mathrm{Pb})$ na ilha de Itacolomi no litoral do Paraná. A análise foi feita a partir da coleta das espécies Anisotremus surinamensis (Bloch, 1791), Holocentrus adscensionis (Osbeck, 1765), Echinometra lucunter (Linnaeus, 1758) e a camada superficial de sedimento marinho em quatro pontos ao redor da ilha de Itacolomi. As amostras foram abertas utilizando a digestão ácida e analisadas em um espectrômetro de absorção atômica. As concentrações médias obtidas no presente estudo foram de $0,435 \mu \mathrm{g} \cdot \mathrm{g}^{-1}$ para o $\mathrm{Cd}, 0,296 \mu \mathrm{g} \cdot \mathrm{g}^{-1}$ para o Hg; o As não foi detectado e o $\mathrm{Pb}$ ficou abaixo do limite de quantificação para o tecido muscular da espécie $H$. adscensionis. Para a espécie $A$. surinamensis, as concentrações médias foram de 0,785 $\mu \mathrm{g} . \mathrm{g}^{-1}$ para o As, enquanto para os metais $\mathrm{Cd}, \mathrm{Hg}$ e $\mathrm{Pb}$, as médias ficaram abaixo do limite de quantificação, também para o tecido muscular. No ouriço do mar E. lucunter o tecido gonadal apresentou as médias de $6,118 \mu \mathrm{g} \cdot \mathrm{g}^{-1}$ para o As, 0,205 $\mu \mathrm{g} \cdot \mathrm{g}^{-1}$ para o $\mathrm{Hg}$, enquanto o $\mathrm{Cd}$ e o $\mathrm{Pb}$ apresentaram médias abaixo do limite de quantificação. $\mathrm{Na}$ análise da presença de metais pesados no sedimento superficial, ficou evidenciado um valor médio de $0,5047 \mu \mathrm{g} \cdot \mathrm{g}^{-1}$ para o $\mathrm{Cd}$, valor médio de $3,2752 \mu \mathrm{g} \cdot \mathrm{g}^{-1}$ para o As, $0,2235 \mu \mathrm{g} \cdot \mathrm{g}^{-1}$ para o $\mathrm{Hg}$ e $0,4038 \mu \mathrm{g} \cdot \mathrm{g}^{-1}$ para o $\mathrm{Cu}$. Os resultados apresentados neste

1 Me.; Gestão Ambiental; Universidade Positivo; Endereço: Organização Paranaense de Ensino Técnico Ltda, Faculdade Organização Paranaense de Ensino Técnico Ltda. Avenida Iguaçú, 755, Rebouças; CEP:80230-020 - Curitiba, PR Brasil; E-mail: renetsantos@yahoo.com.br

2 Me.; Química; Universidade Estadual Paulista Júlio de Mesquita Filho, UNESP, Brasil; Professor adjunto da Universidade Positivo; Universidade Positivo. R.: Prof. Pedro Viriato Parigot de Souza, 5300 - Núcleo de Controle de Qualidade e Desenvolvimento, Campo Comprido. CEP: 81280330 - Curitiba, PR - Brasil; E-mail: fcneto@up.edu.br

3 Dr.; Zoologia; Universidade Federal do Paraná; Endereço profissional: Rua Jaguariaíva, 512 - Caiobá, Matinhos - PR, CEP: 83260-000 E-mail: edinaoli@yahoo.com

4 Dr.; Química; Universidade Estadual Paulista Júlio de Mesquita Filho, UNESP, Brasil; Professor da Universidade Positivo no Parana, atuando nos Programas de Doutorado e Mestrado em Gestão Ambiental e Mestrado em Biotecnologia; Endereço: Universidade Positivo, Pró Reitoria de Pós Graduação e Pesquisa. Rua Prof Pedro Viriato Parigot de Souza, 5300 Coordenação de Pesquisa, Campo Comprido. CEP: 81280-330 - Curitiba, PR - Brasil; E-mail: mcarvalho@up.edu. br

5 Dra.; Química; Universidade de São Paulo, USP, Brasil; Professor titular da Universidade Positivo; Endereço: Centro Universitário Positivo, Mestrado Profissionalizante em Gestão Ambiental. R. Pedro Viriato Parigot de Souza, 5300, Campo Comprido. CEP: 81280-330 - Curitiba, PR - Brasil; E-mail: evasconcelos@up.edu.br

\begin{tabular}{llllll}
\hline Ambiência & Guarapuava (PR) & v.14 n.3 & p.461-476 & Set/Dez 2018 & ISSN $1808-0251$
\end{tabular}


trabalho, demonstraram a presença de metais pesados abaixo dos limites estabelecidos para consumo humano ( $\mathrm{Cd}$ e As= $\left.1 \mu \mathrm{g} \cdot \mathrm{g}^{-1} ; \mathrm{Hg}=0,5 \mu \mathrm{g} \cdot \mathrm{g}^{-1} ; \mathrm{Pb}=2 \mu \mathrm{g} \cdot \mathrm{g}^{-1}\right)$.

Palavras-chave: Cádmio; Chumbo; Mercúrio; Arsênio; Poluição ambiental; Biota.

\section{Abstract}

The analyses were made from the collection of species $A$. surinamensis, $H$. adscensionis, $E$. lucunter and the superficial layer of marine sediment at four points around the Itacolumi Island. The mean concentrations obtained in this study were $0.435 \mu \mathrm{g} \cdot \mathrm{g}^{-1}$ for $\mathrm{Cd}, 0.296 \mu \mathrm{g} . \mathrm{g}^{-1}$ for $\mathrm{Hg}$, As and $\mathrm{Pb}$ were not detected for the muscle tissue of the species $H$. adscensionis. For the species $A$. surinamensis, the average concentrations were $0.785 \mu \mathrm{g} . \mathrm{g}^{-1}$ for As, while for the metals $\mathrm{Cd}, \mathrm{Hg}$ and $\mathrm{Pb}$ were detected only. In the sea urchin E. lucunter gonadal tissue was analyzed and showed the averages of $6.118 \mu \mathrm{g} \cdot \mathrm{g}^{-}$ ${ }^{1}$ for $\mathrm{As}, 0.205 \mu \mathrm{g} \cdot \mathrm{g}^{-1}$ for $\mathrm{Hg}, \mathrm{Cd}$ and $\mathrm{Pb}$ were detected only. In the analysis of heavy metals in sediment surface evidenced a mean value of $0.5047 \mu \mathrm{g} \cdot \mathrm{g}^{-1}$ to $\mathrm{Cd}, 3.2752$ $\mu \mathrm{g} \cdot \mathrm{g}^{-1}$ average value for $\mathrm{As}, 0.2235 \mu \mathrm{g} \cdot \mathrm{g}^{-1}$ for $\mathrm{Hg}$ and $0.4038 \mu \mathrm{g} \cdot \mathrm{g}^{-1}$ for $\mathrm{Cu}$. The results presented in this study demonstrated the presence of heavy metals below allowed for human consumption.

Keywords: Environmental pollution, E. lucunter, H. adscensionis, A. surinamensis.

\section{Introdução}

Muitas vezes, a poluição nos sistemas ambientais tem sua origem em fontes locais como também fontes não locais, o que pode promover a degradação dos sistemas ecológicos dos oceanos, costas e mares, tornando-se um problema ambiental de dimensões intercontinentais (MAANAN, 2008; GUSSO-CHOUERI et al., 2018; BONSIGNORE et al., 2018).

Alguns autores entendem que uma grande parte da poluição dos oceanos e mares tem sua origem na disseminação por fontes terrestres, basicamente industriais e urbanas (GOULART; CALLIXTO, 2003; MACÍAZ-ZAMORA e RAMIREZ-ALVAREZ, 2004, WANG, et al., 2012). Turner, 2010, diz que a contaminação de águas e sedimentos marinhos por $\mathrm{Ba}, \mathrm{Cd}, \mathrm{Cu}, \mathrm{Ni}$ $\mathrm{Pb}$ e $\mathrm{Sn}$ pode estar relacionada às partículas de tinta anti-incrustante, de regiões de manutenção de barcos e galpões abandonados como também de entradas para navios, podendo assim, através de variáveis ambientais acumularem em invertebrados marinhos.

Nos ecossistemas aquáticos, uma grande variedade de organismos, tais como peixes e mariscos que tem capacidade de reter metais pesados, tem sido amplamente utilizado como bioindicadores na intenção de monitorar áreas com alterações nos níveis de poluição (VIEIRA; SHIBATTA, 2007; BONSIGNORE et al.2018; ZHANG et al.2018). Como esses organismos fazem parte da alimentação humana, conhecer os níveis de contaminação de metais é fundamental para se estabelecer a segurança alimentar (MAHECHA, 2002; BURGER; GOCHFELD, 2005; GUSSO-CHOUERI et al. 2018; SOBIHAH et al. 2018). 
O monitoramento de regiões costeiras, usando organismos que possuem a capacidade de acumular metais tem sido utilizado para acompanhar a evolução da poluição nessas regiões (FRANÇA et al., 2005; ONEN, et al. 2011; BONSIGNORE et al. 2018; GUSSO-CHOUERI et al.2018). Os organismos escolhidos precisam ser endêmicos para que se possa ter uma resposta o mais próxima do real possível (ONEN, et al. 2011).

Cádmio $(\mathrm{Cd})$, cobre $(\mathrm{Cu})$, chumbo $(\mathrm{Pb})$ e mercúrio $(\mathrm{Hg})$ são metais que têm sido monitorado em regiões costeiras devido à ligação direta com áreas industriais. Estudos in vitro constataram a capacidade de larvas de Ouriço do mar (Paracentrotu slividus, Lmk.) em acumular $\mathrm{Cd}, \mathrm{Pb}$ e $\mathrm{Cu}$ (RADENAC, et al., 2001). Em águas marinhas esses organismos podem entrar em contato com esses e outros metais que estão dissolvidos ou aderidos ao material particulado. Wang et al. (2012) detectaram concentrações elevadas de $\mathrm{Cu}, \mathrm{Pb}, \mathrm{Zn}, \mathrm{Cd}, \mathrm{Hg}$ no material particulado, suspenso nas águas da Baia de Jinzhou.

O aumento desordenado dos núcleos populacionais, das indústrias, do número de veículos automotores, como também de terminais portuários na costa paranaense e catarinense, vem contribuindo para o aumento de emissões de efluentes e gases com os devidos tratamentos, muitas vezes, inadequados ou, até mesmo ineficientes. Esses fatores servem de alerta para que o monitoramento dessa região seja iniciado fim de se estabelecerem parâmetros que possam ser usados no futuro.

O presente trabalho teve como objetivo fazer um diagnóstico inicial da presença de metais pesados nas proximidades da ilha Itacolomi, no litoral do Paraná. Para isso, avaliou-se a presença dos metais pesados ( $\mathrm{Hg}, \mathrm{As}, \mathrm{Cd}$ e $\mathrm{Pb}$ ) nos tecidos musculares dos peixes Anisotremus surinamensis (Bloch, 1791) e Holocentrus adscensionis (Osbeck, 1765); do tecido gonadal do equinoderma Echinometra lucunter (Linnaeus, 1758), como também, a presença dos metais pesados ( $\mathrm{Hg}, \mathrm{As}, \mathrm{Cd}$ e $\mathrm{Cu}$ ) na camada superficial do sedimento marinho da ilha de Itacolomi, Estado do Paraná, Brasil.

\section{Materiais e Métodos}

\section{Área de estudo}

A área de estudo compreende a ilha de Itacolomi, litoral do Paraná, com sua posição geográfica para o Rochedo Leste a $25^{\circ} 50^{\prime} 32,87^{\prime \prime} \mathrm{S}, 48^{\circ} 24^{\prime 2} 25,46^{\prime \prime} \mathrm{O}$ e para o Rochedo Oeste 2550'30,34” S, 48²4'29,73” O, sendo os rochedos mais distantes do litoral do Paraná (DAROS, 2010; PORTAL OFICIAL DO MUNICÍPIO DE GUARATUBA, 2011).

A ilha está situada há 7,3 milhas náuticas da entrada da baía de Guaratuba, cidade litorânea, distante 119 quilômetros de Curitiba, capital do Estado do Paraná, Brasil, apresenta uma distância

ainda, de 7 milhas náuticas das ilhas de Currais (Figura 1), a profundidade da região no entorno da ilha foi demonstrado pela batimetria entre 3 e 17 metros (DAROS, 2010; PORTAL OFICIAL DO MUNICÍPIO DE GUARATUBA, 2011). 
Figura 1- Imagem das ilhas de Itacolomi e Currais e sua localização no litoral Paranaense.

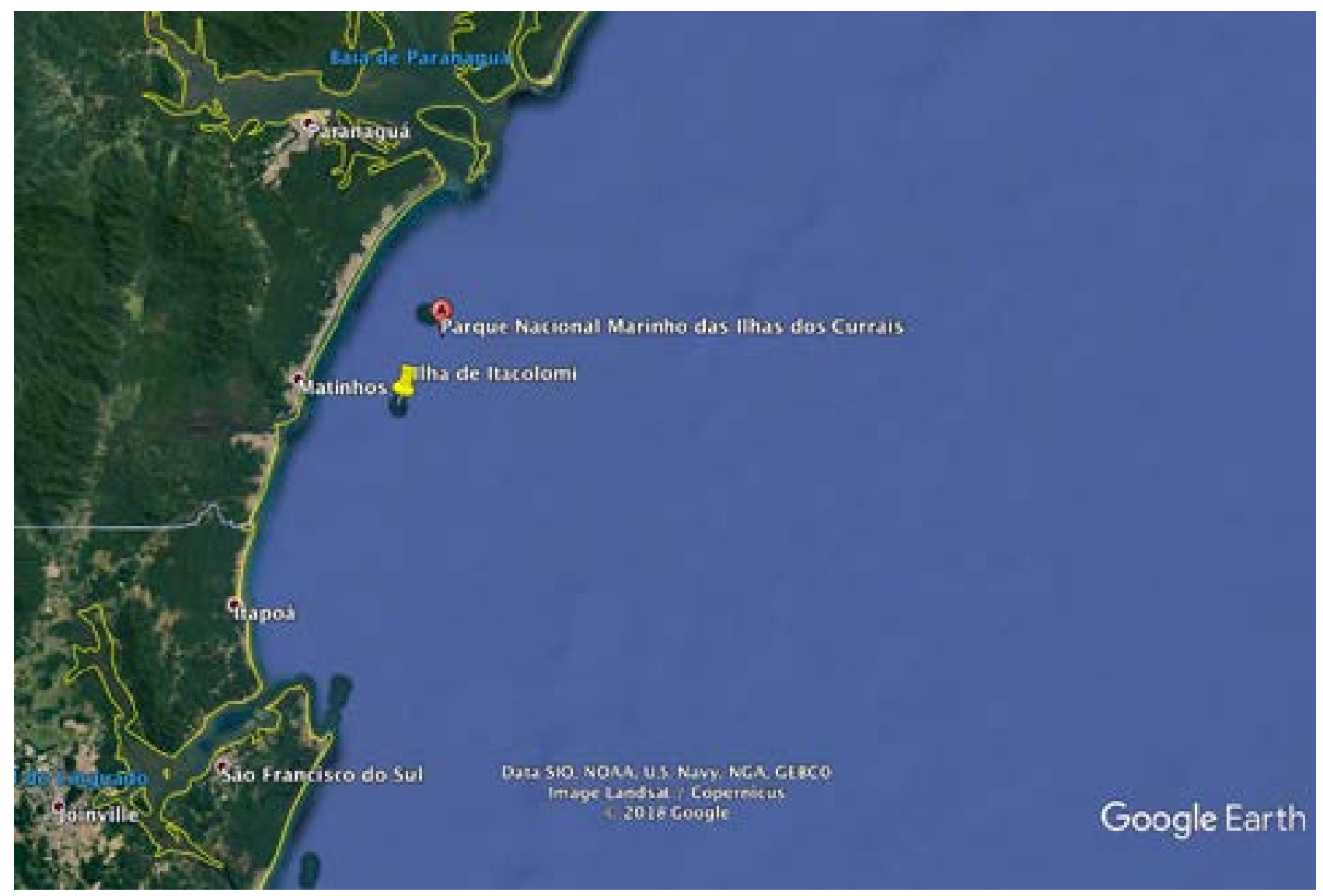

Coleta das amostras

Para o desenvolvimento deste trabalho, foram determinados 04 sítios para coleta das

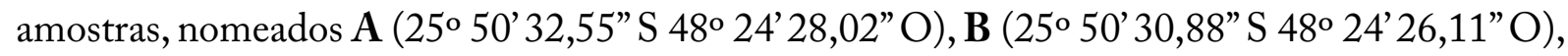

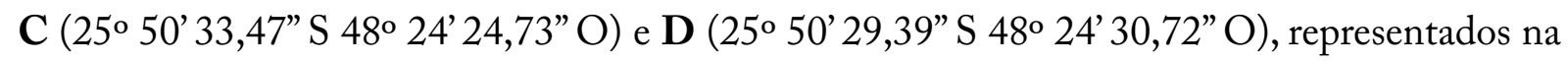
Figura 2. 


\section{Figura 2. Imagem dos rochedos oeste e leste com os sítios de coleta na Ilha de Itacolomi.}

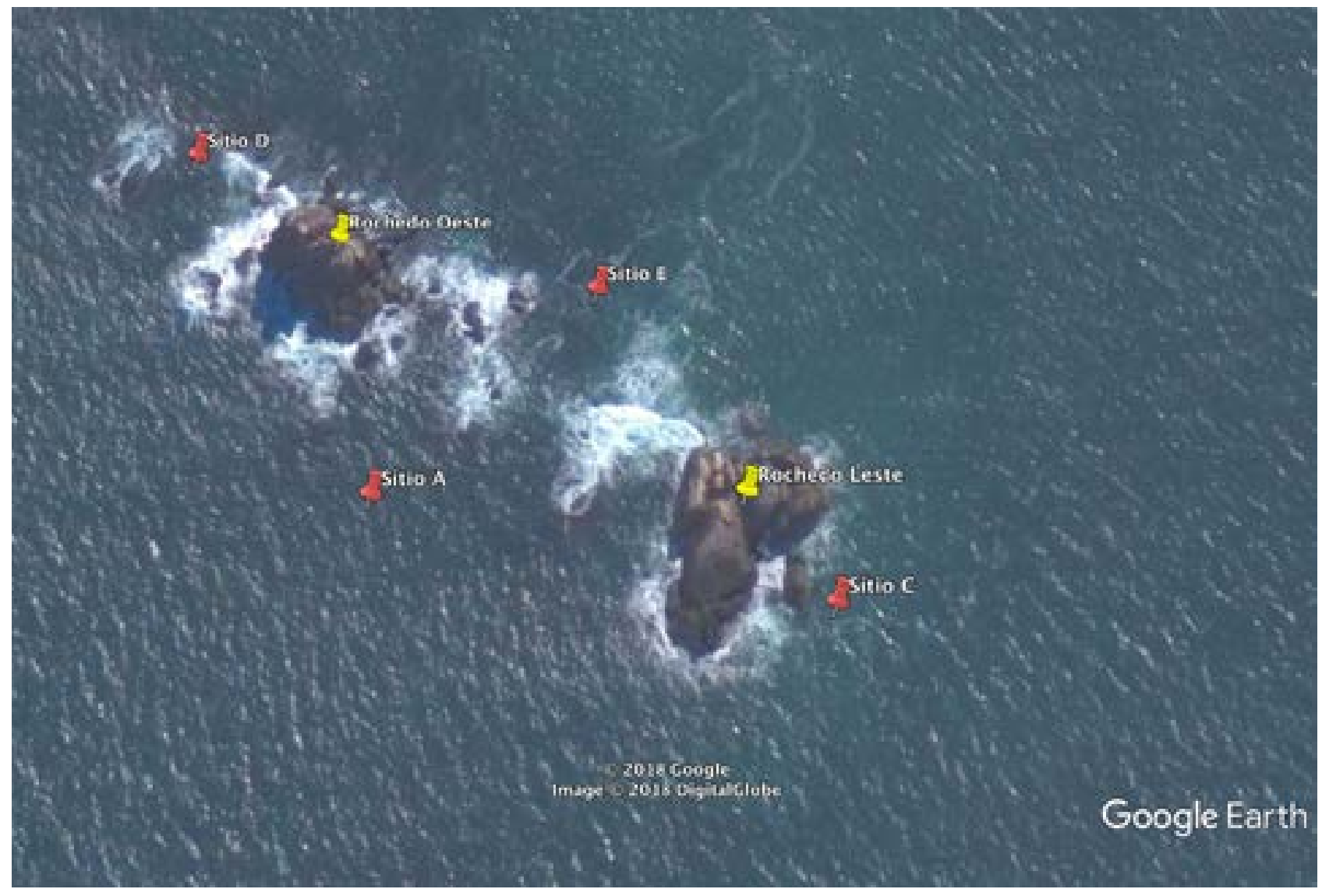

\section{Peixes}

Foram utilizados os peixes Anisotremus surinamensis (Bloch,1791) e Holocentrus adscensionis (Osbeck, 1765), sendo abatidos através de tiro de arbalete no período de 21 e 22 de abril de 2011. Foram coletados seis exemplares de cada espécie, em cada um dos quatro sítios em torno da ilha de Itacolomi, perfazendo um total de 24 exemplares por espécie, sem discriminação de sexo. Os exemplares foram abertos com o uso de faca de aço inox para a separação do tecido muscular; uma vez separados, os tecidos musculares foram congelados à temperatura de $-10^{\circ} \mathrm{C}$.

\section{Ouriço do mar}

Ouriço do mar Echinometra lucunter (Linnaeus, 1758), foram coletados por retirada manual do fundo rochoso onde se encontravam. Foram coletados seis exemplares por sítio de coleta, totalizando 24 exemplares. Os exemplares foram abertos com o uso de serra fina para metal, cortando-se na circunferência maior do esqueleto, sendo, em seguida, retirado o tecido gonadal e alojado em 04 placas de Petri; foram pesados e transferidos para o congelamento à temperatura de $-10^{\circ} \mathrm{C}$. 
O tecido muscular dos peixes e o tecido gonadal dos ouriços do mar foram liofilização à $-55^{\circ} \mathrm{C}$, utilizando o liofilizador da marca Ílshin Lab Co., Ltda.

\section{Sedimento}

A coleta do sedimento foi efetuada à profundidade dos 10 metros entre as rochas e o sedimento, através de coleta manual. $\mathrm{O}$ sedimento foi apanhado utilizando colher de plástico e depositado em saco plástico do tipo Zip Lock furado na base para escoar o excesso de água.

Em seguida foi selado com plástico do tipo filme e colocado em recipiente com gelo, sendo transportado para o laboratório em câmara térmica, onde foi aberto após pesagem total do conteúdo do saco plástico e descontado o valor do peso do saco plástico.

Foram separadas 04 amostras, com peso de 642,82 gramas para a amostra do sítio A, 758,91 gramas para a amostra do sítio B, 456,14 gramas para a amostra do sítio C e 641,38 gramas para a amostra do sítio $\mathrm{D}$, para o estudo de determinação granulométrica no laboratório de Mecânica dos Solos da Universidade Positivo.

O restante do material foi encaminhado para o laboratório de águas do Núcleo de Controle de Qualidade e Desenvolvimento na Universidade Positivo, onde foi feita a verificação da presença de metais pesados através da análise de determinação de metais em sedimentos por espectrofotometria de absorção atômica da CETESB (1994).

\section{Extração e análise dos metais ( $\mathrm{Pb}, \mathrm{Cd}, \mathrm{Hg}, \mathrm{As}$ e $\mathrm{Cu})$}

Para abertura das amostras, foi utilizado o método de digestão ácida conforme descrito pelos métodos de determinação de metais em peixes e animais aquáticos após liofilização e em sedimentos e solo da Companhia de Tecnologia de Saneamento Ambiental do Estado de São Paulo (CETESB, 1994), com o embasamento no Standard Methods for the Examination of Water and Wastewater - (APHA, 1998). Pesou-se $\pm 2,0 \mathrm{~g}$ de tecido muscular e, após o procedimento de digestão a solução, foi avolumada a $50 \mathrm{~mL}$. Para análise do tecido gonadal pesou-se $\pm 0,5 \mathrm{~g}$ e o volume final da solução foi de $50 \mathrm{~mL}$. Para o sedimento, pesou-se $\pm 2,0 \mathrm{~g}$ e o volume final foi de $50 \mathrm{~mL}$.

Após a abertura das amostras, os extratos foram analisados utilizando-se um Espectrômetro de Absorção Atômica (EAA) da marca Shimadzu, modelo AA 6800. A análise do chumbo foi realizada utilizando-se EAA por chama com forno de grafite - SM 3111 B.

Os valores obtidos foram submetidos ao teste $t$ de Student utilizando-se a ferramenta XLSTAT versão 2011 do Excell, onde os resultados foram comparados dois a dois.

\section{Resultados e Discussão}

\section{Concentração dos metais nos organismos avaliados}

Os valores obtidos para os metais presentes nos organismos avaliados foram comparados aos valores de referência do órgão brasileiro regulamentador, Agencia Nacional de Vigilância Sanitária (ANVISA), Portaria n 685, de 27 de agosto de 1998 (BRASIL, 1998) para a presença desses metais em peixes e derivados da pesca. 
A Tabela 1 apresenta as concentrações dos metais $\mathrm{Cd}, \mathrm{As}, \mathrm{Hg}$ e $\mathrm{Pb}$ encontradas nas amostras de tecido muscular do peixe Holocentrus adscensionis (Osbeck, 1765).

\begin{tabular}{cccccc} 
Tabela 1 - Concentração $\left(\boldsymbol{\mu g . g ^ { - 1 } )}\right.$ dos metais $\mathbf{C d}$, As, Hg e Pb \\
encontradas no tecido muscular do peixe Holocentrus \\
adscensionis (Osbeck, 1765) \\
\hline Metal & Sítio A & Sítio B & Sítio C & Sítio D & V.R. \\
\hline $\mathrm{Cd}$ & $0.583 \pm 0.390$ & $0.220 \pm 0.013$ & $0.327 \pm 0.566$ & $0.610 \pm 0.816$ & 1 \\
$\mathrm{As}$ & N.D. & N.D. & N.D. & N.D. & 1 \\
$\mathrm{Hg}$ & N.D. & N.D. & $0.255 \pm 0.058$ & $0.337 \pm 0.094$ & 0.5 \\
$\mathrm{~Pb}$ & A.L.Q. & A.L.Q. & A.L.Q. & A.L.Q. & 2 \\
\hline
\end{tabular}

N.D. = Não Detectado

A.L.Q. = Abaixo do Limite de Quantificação

V.R. = Valor de Referência proposto pela Portaria $n^{\circ}$ 685/98 ANVISA

As concentrações obtidas para o $\mathrm{Cd}$ não apresentaram diferença significativa entre os sítios amostrados, de acordo com o teste $t$ de Student para duas populações ( $p<0,05=$ diferença significativa). As mesmas variaram entre 0,220 e $0,610 \mu \mathrm{g} \cdot \mathrm{g}^{-1}$ e ficaram abaixo do limite estabelecido Portaria $n^{\circ}$ 685/98 da ANVISA.

O mercúrio não foi identificado nos sítios $\mathrm{A}$ e $\mathrm{B}$ e as concentrações obtidas para os sítios $\mathrm{C}$ e D não apresentaram diferença significativa entre os dois pontos. $\mathrm{O}$ mercúrio apresentou o valor médio de 0,296 $\mu \mathrm{g} \cdot \mathrm{g}^{-1}$, sendo abaixo do valor recomendado pela Portaria n ${ }^{\circ}$ 685/98 da ANVISA.

$\mathrm{O}$ arsênio não foi detectado nas amostras de tecido muscular do $H$. Adscensionis.

O chumbo foi detectado, porém ficou abaixo do limite de quantificação, não possibilitando, assim, determinar seu real valor.

A Tabela 2 apresenta as concentrações dos metais $\mathrm{Cd}, \mathrm{As}, \mathrm{Hg}$ e $\mathrm{Pb}$ encontradas nas amostras de tecido muscular do peixe Anisotremus surinamensis (Bloch, 1791).

\section{Tabela 2 - Concentração $\left(\mu \mathrm{g} \cdot \mathrm{g}^{-1}\right)$ dos metais $\mathrm{Cd}, \mathrm{As}, \mathrm{Hg}$ e $\mathrm{Pb}$ encontradas no tecido muscular do peixe Anisotremus surinamensis (Bloch, 1791)}

\begin{tabular}{cccccc}
\hline Metal & Sítio A & Sítio B & Sítio C & Sítio D & V.R. \\
\hline $\mathrm{Cd}$ & A.L.Q. & A.L.Q. & A.L.Q. & A.L.Q. & 1 \\
$\mathrm{As}$ & $1.25 \pm 0.014$ & $0.75 \pm 0.014$ & $0.69 \pm 0.084$ & $0.45 \pm 0.327$ & 1 \\
$\mathrm{Hg}$ & A.L.Q. & A.L.Q. & A.L.Q. & A.L.Q. & 0.5 \\
$\mathrm{~Pb}$ & A.L.Q. & A.L.Q. & A.L.Q. & A.L.Q. & 2 \\
\hline
\end{tabular}

A.L.Q. = Abaixo do Limite de Quantificação

V.R. = Valor de Referência proposto pela Portaria $n^{\circ}$ 685/98

As concentrações obtidas para o As apresentaram diferenças significativas entre os sítios A e B ( $p=0.001)$, A e C ( $p=0.012)$ e A e D ( $p=0.047)$, de acordo com o teste $t$ de Student para 2 populações. Não houve diferença significativa entre os sítios $\mathrm{B}, \mathrm{C}$ e D. As concentrações variaram entre 0,45 e $1,25 \mu \mathrm{g} \cdot \mathrm{g}^{-1}$. 
$\mathrm{Cd}, \mathrm{Hg}$ e $\mathrm{Pb}$ foram detectados, mas os valores detectados ficaram abaixo do limite de quantificação, o que pode ser sugestivo de que esses valores, apesar de estarem abaixo dos limites devam ser melhor avaliados com níveis de quantificação menores.

$\mathrm{Na}$ Tabela 3, apresentam-se as médias dos valores detectados nas amostras de tecido gonadal do equinoderma Echinometra lucunter (Linnaeus, 1758) nos sítios avaliados.

\section{Tabela 3 - Concentração ( $\mu$ g.g-1) dos metais Cd, As, $\mathrm{Hg}$ e Pb encontradas no tecido gonadal do equinoderma Echinometra lucunter ( Linnaeus, 1758)}

\begin{tabular}{cccccc}
\hline Metal & Sítio A & Sítio B & Sítio C & Sítio D & V.R. \\
\hline $\mathrm{Cd}$ & A.L.Q. & A.L.Q. & A.L.Q. & A.L.Q. & 1 \\
$\mathrm{As}$ & $6.92 \pm 1.357$ & $5,65 \pm 0.606$ & $6.243 \pm 2,477$ & $5,660 \pm 0.588$ & 1 \\
$\mathrm{Hg}$ & $0.193 \pm 0.105$ & $0.195 \pm 0.148$ & $0.195 \pm 0.007$ & $0.240 \pm 0.070$ & 0.5 \\
$\mathrm{~Pb}$ & A.L.Q. & A.L.Q. & A.L.Q. & A.L.Q. & 2 \\
\hline
\end{tabular}

A.L.Q. = Abaixo do Limite de Quantificação

V.R. $=$ Valor de Referência proposto pela Portaria $n^{\circ} 685 / 98$

As concentrações obtidas para o As e $\mathrm{Hg}$ não apresentaram diferenças significativas entre os sítios A B, C e D, em acordo com o teste t de Student. Cd e Pb foram detectados, porém estavam abaixo do limite de quantificação.

O As apresentou um valor seis vezes acima do valor de referência para derivados de pesca, variando de 5.65 a $6.92 \mu \mathrm{g} \cdot \mathrm{g}^{-1}$.

$\mathrm{O} \mathrm{Hg}$ apresentou valores que ficaram entre 0.192 e $0.240 \mu \mathrm{g} \cdot \mathrm{g}^{-1}$, valores que estão abaixo do limite de $0.5 \mu \mathrm{g}$.g-1 estabelecido pela resolução.

Até o presente momento, a análise do tecido muscular da amostra Holocentrus adscensionis (Osbeck, 1765) e da amostra Anisotremus surinamensis (Bloch, 1791) evidencia uma concentração dos metais pesados mercúrio, arsênio, cádmio e chumbo abaixo dos limites recomendados para consumo humano de tecido muscular de peixes e frutos do mar pela Portaria ${ }^{\circ}$ 685/98 (BRASIL, 1998).

Porém, a maior concentração de arsênio encontrada no tecido muscular do Anisotremus surinamensis (Bloch, 1791) pode estar relacionada a sua dieta alimentar a qual inclui o ouriço do mar Echinometra lucunter (Linnaeus, 1758). Todos os exemplares de Echinometra lucunter (Linnaeus, 1758), pescados para a realização do presente trabalho, continham, em sua boca, restos de ouriço do mar. Essa relação pode ser proposta em virtude das altas concentrações de arsênio encontradas no tecido gonadal do Echinometra lucunter (Linnaeus, 1758) aqui analisados, Tabela 3 , seis vezes acima do valor de referência.

A razão para a diferença entre os pontos de amostragem para o As precisa ser melhor avaliada, podendo estar relacionadas às correntes marinhas, porém, para tal hipótese ser considerada, seria necessário um estudo mais aprofundado dessas correntes, estudo este não realizado no presente trabalho.

Podemos sugerir que o Anisotremus surinamensis (Bloch, 1791), devido a seu hábito alimentar estar incluso o ouriço do mar, ainda pode ser um bom bioindicador para o As.

$\mathrm{O}$ acumulo de As seis vezes maior do que o valor de referência no ouriço do mar pode estar ligado a existência natural de arsênio nas rochas e a sua ampla distribuição na superfície 
marinha. Outro fato é que o ouriço do mar tem, em seu hábito alimentar, a escavação nas rochas através de seu sistema bucal onde seu comportamento perfurador parece ser uma adaptação para compensar a ação das correntes marítimas (BRUSCA e BRUSCA, 2007). Esse fato pode ser melhor evidenciado pelas concentrações de As encontradas no sedimento (Tabela 4). Embora as concentrações estejam dentro do mínimo aceitável para não causar efeito na biota, o As pode estar sendo incorporado pelo ouriço do mar. O ouriço juntamente com o Anisotremus surinamensis poderiam ser monitorados para se verificar como essas concentrações estariam evoluindo ao longo do tempo. Resultado semelhante foi observado por Zhang et al. (2018) quando avaliaram organismos com hábitos alimentares iguais aos do ouriço e $A$. surnamensis.

O mercúrio apresentou uma distribuição irregular nas amostras. Dentre os espécimes avaliados o ouriço do mar apresentou contaminação pelo mercúrio em todas as amostras. Esse processo pode estar relacionado aos seu habito alimentar. Relacionando esses dados com o resultado encontrado para o mercúrio no sedimento podemos sugerir que está havendo uma influência do mercúrio encontrado no sedimento nessa concentração. Fato preocupante, pois todas as amostras apresentaram valores acima do valor de referência. Embora o mercúrio não tenha sido quantificado nas amostras de Anisotremus surinamensis, ele foi detectado. A presença de mercúrio no sedimento, na biota ou mesmo na coluna de água pode estar relacionada a diversos fatores como despejos de esgotos, atividade industrial ou mesmo eventos naturais (erupção vulcânica) (NRIAGU and BECKER, 2003; SIQUEIRA et al, 2005; ULUTURHAN et al, 2011; BUBACH et al, 2012).

A distribuição de mercúrio na cadeia trófica é um fato conhecido, com tendência de decrescer a concentração na ordem carnívoros > onívoros > herbívoros > detritívoros (MORGANO et al, 2007). Por isso a importância de monitorar a presença desse metal no ambiente marinho. Uma vez presente ele pode contaminar peixes e outras espécies que fazem parte da cadeia alimentar humana.

Morgano et al (2007) encontraram na baixada Santista valores dentro do permitido para os pescados não-predadores e valores acima do permitido para os predadores, reafirmando o processo de acumulo do mercúrio.

Peixes e outras espécies de hábitos locais não migratórios e detritívoros e/ou herbívoros são importantes bioindicadores de contaminação por metais pesados (ONEN et al, 2011).

Embora os valores de $\mathrm{Cd}$ encontrados no tecido muscular do H. adscensionis, apresentem-se dentro dos limites para o consumo humano de acordo com a Portaria n ${ }^{\circ}$ 685/98 da ANVISA, esses valores estão acima de valores encontrados por outros autores que avaliaram espécies semelhantes, que se comportam de maneira sedentária. $\mathrm{O} \mathrm{Cd}$ não foi quantificado nas outras duas espécies avaliadas, mas foram detectados denotando a sua presença no ambiente. Turkmen et al. (2005), Dalman et al. (2006), Chi et al. (2007) e Sobihah et al. (2018) encontraram valores que variaram de 0,01 a $0,047 \mu \mathrm{g} \cdot \mathrm{g}^{-1} \mathrm{de} \mathrm{Cd}$ nas espécies que foram avaliadas por esses autores. A ocorrência de $\mathrm{Cd}$ no ambiente marinho está muito relacionada a atividade industrial. Amado - Filho et al. (2008) analisaram a presença de metais em quatro espécies de organismos marinhos bentônicos (Padina gymnospora, Sargassum sp., Halodule wrightii e Crassostrea rhizophorae) e em três sítios de amostragem na região mais afetada por atividades industriais, encontraram valores elevados de $\mathrm{Cd}$ em todas as espécies. Em regiões com forte atividade industrial próximas a baias e estuário podem apresentar valores de concentração de cádmio maiores do que as encontradas no presente trabalho (AMADO-FILHO et al, 2008). 
O chumbo não apresentou concentração suficiente nos organismos analisados para ser quantificada $\left(>1 \mu \mathrm{g} \cdot \mathrm{g}^{-1}\right.$ ), mas foi detectado. A presença de chumbo pode estar associada a forte atividade de pesca amadora e de subsistência de ocorre ao redor da ilha. Durante o mergulho para pesca submarina é possível ver artefatos utilizados pelos pescadores depositados sobre o sedimento. Tal fato pode estar relacionado as concentrações de chumbo que foram determinadas para o sedimento.

\section{Concentração dos metais no sedimento}

Importante levar em conta na análise do sedimento suas características granulométricas, onde existe uma predisposição na adsorção de metais pesados pelos grânulos finos, como areia muito fina o silte e a argila.

Para os autores Rubio et al. (2000) e Otim et al. (2018) o diâmetro da partícula do sedimento tem suma importância na afinidade dos metais pesados, onde os grãos de diâmetros de areia muito fina $(0,125$ a 0,062$)$, silte $(0,062$ a 0,00394$)$ e argila $(0,00394$ a 0,0002$)$ tem maior afinidade por metais pesados, adsorção.

O sedimento analisado apresentou granulometria variando de areia muito fina a cascalho fino, Figura 3. A maior parte do sedimento é composto de areia média e fina, as quais apresentaram porcentagens muito próximas. Os resultados demonstram uma média baixa de areia muito fina, $0,78 \%$, o que pode levar a interpretar que os valores dos metais pesados $\mathrm{Cd}, \mathrm{Cu}, \mathrm{As}$ e $\mathrm{Hg}$ apresentados podem não revelar a real concentração ou disponibilidade destes metais na região pela análise granulométrica.

Figura 3 - Granulometria do sedimento retirado da Ilha de Itacolomi nos sítios A, B, C e D. Em destaque areia muito fina.

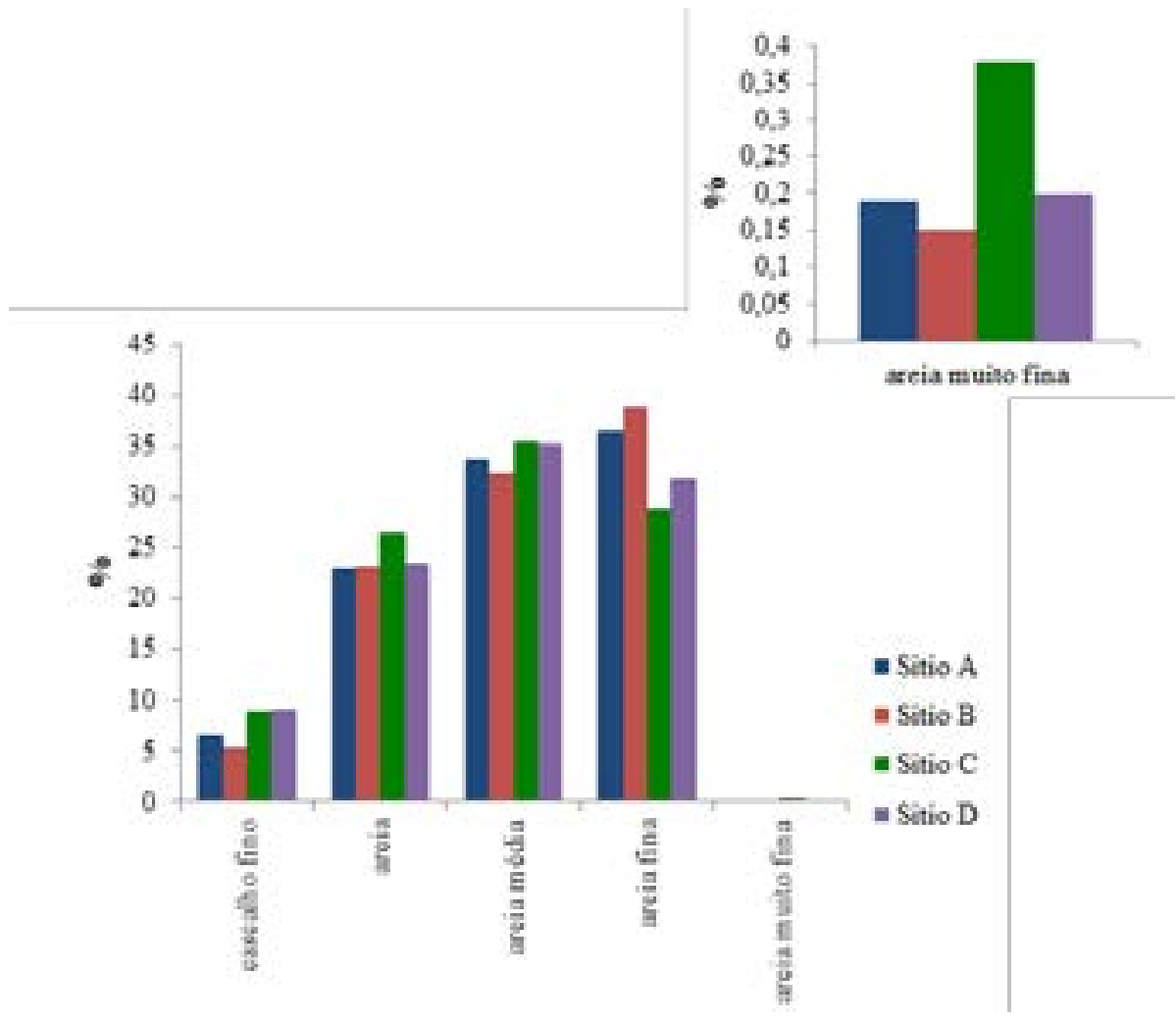


A Tabela 4 apresenta as concentrações dos metais pesados $\mathrm{Cd}, \mathrm{Cu}, \mathrm{As}$ e $\mathrm{Hg}$ determinadas nas amostras de sedimento.

\section{Tabela 4 - Concentração $(\mu \mathrm{g} . \mathrm{g}-1)$ de metais encontrados para o sedimento coletado na ilha de Itacololmi.}

\begin{tabular}{ccccc}
\hline Metal & Sítio A & Sítio B & Sítio C & Sítio D \\
\hline $\mathrm{Cd}$ & $0.761 \pm 0.259$ & $0.336 \pm 0.260$ & $0.534 \pm 0.484$ & $0.387 \pm 0.0 .179$ \\
$\mathrm{As}$ & $5.590 \pm 2.270$ & $2.458 \pm 1.283$ & $1.613 \pm 0.631$ & $3.440 \pm 0.534$ \\
$\mathrm{Hg}$ & $0.335 \pm .084$ & $0.153 \pm 0.018$ & $0.141 \pm 0.015$ & $0.265 \pm 0.036$ \\
$\mathrm{Cu}$ & $0.790 \pm 0.042$ & $0.650 \pm 0.111$ & $0.089 \pm 0.006$ & $0.086 \pm 0.005$ \\
\hline
\end{tabular}

Os valores obtidos para o Cd não apresentaram diferença significativa entre eles de acordo com o teste $\mathrm{t}$ de Student para as populações, já as concentrações obtidas de $\mathrm{Cu}$ apresentaram diferença significativa entre os sítios A e C ( $\mathrm{p}=0.043)$ e os sítios C e D ( $\mathrm{p}=0.019)$. As concentrações obtidas de As apresentaram diferença significativa entre os sítios A e B ( $p=0.021)$, sítios A e $C$ ( $p=0.017)$, sítios B e D ( $p=0.009)$ e sítios C e D ( $\mathrm{p}=0.005)$. As concentrações obtidas de $\mathrm{Hg}$ apresentaram diferença significativa entre os sítios A e C ( $p=<0.0001)$, sítios A e D ( $p=<0.0001)$, sítios B e C ( $\mathrm{p}=0.001)$ e sítios B e D ( $\mathrm{p}=0.001)$.

Não existe na legislação Brasileira uma resolução específica para a contaminação de sedimentos que pudesse ser utilizada para comparação. Para tanto utilizou-se os valores de referência preconizados na Resolução CONAMA 344/04 que trata da qualidade de sedimentos oriundos de dragagem em águas salinas ou salobras, Quadro 1, na resolução o nível de metal pesado no sedimento pode ser classificado em nível 1 e 2 para água doce e nível 3 e 4 para água salina. $\mu g . g-1$.

\section{Quadro 1 - Valores de referência em $\mu$ g.g-1 preconizados na Resolução CONAMA 344/04 para concentração de metais em sedimento}

\begin{tabular}{|c|c|c|c|c}
\hline Metal & Nivel 1 & Nivel 2 & Nivel 3 & Nivel 4 \\
\hline Cd & 0,6 & 3,5 & 1,2 & 9,6 \\
$\mathrm{Cu}$ & 35,7 & 197 & 34 & 270 \\
$\mathrm{As}$ & 5,9 & 17 & 8,2 & 70 \\
$\mathrm{Hg}$ & 0,17 & 0,486 & 0,15 & 0,71 \\
\hline
\end{tabular}

O nível 3 representa limiar do qual se prevê baixa probabilidade de efeitos adversos à biota O nível 4 representa limiar do qual se prevê um provável efeito adverso à biota

$\mathrm{O}$ arsênio apresentou valores que variaram de 1,613 a 5,590 $\mu \mathrm{g} \cdot \mathrm{g}^{-1}$, o resultado ficou abaixo dos valores recomendados pela resolução CONAMA 344/04, que é para o nível 3 de 8,2 $\mu \mathrm{g} \cdot \mathrm{g}^{-1}$.

$\mathrm{O}$ mercúrio apresentou valores que variaram de 0,141 a $0,335 \mu \mathrm{g} \cdot \mathrm{g}-1$. Os sítios $\mathrm{B}$ e $\mathrm{C}$ atendem a resolução ficando abaixo do nível 3. Porém os sítios A e D estão entre os níveis 3 e 4, o que sugere uma maior investigação pois as concentrações encontradas podem acarretar em efeitos adverso a biota de acordo com a resolução CONAMA 344/04 (BRASIL, 2014).

Uma possível causa para as diferenças nas concentrações dos metais pode estar nas correntes marinhas, porém para se chegar a uma conclusão considerando esta hipótese seria necessário estudo mais aprofundado dessas correntes, estudo esse não realizado no presente trabalho. 
As condições físico-químicas também exercem forte ação sobre a deposição de metais no sedimento de fundo. A deposição em ambientes aquáticos é facilitada em direção aos ambientes marinhos, ou seja, aumentado da foz para o alto mar (SIQUEIRA e APRILE, 2012; OTIM et al., 2018). Porém, esse processo pode sofrer influência de transição dos rios e o oceano. O litoral do Paraná não possuiu nenhum sistema de foz que provoque grandes alterações nas concentrações de sais ou no valor de $\mathrm{pH}$ em virtude da mistura da água doce com a salgada. Situação observada na região de transição entre a foz dos rios Amazonas e Pará e o oceano (SIQUEIRA e APRILE, 2012). A proximidade com a foz de rios influencia a precipitação e a retenção no sedimento de fundo devido a maior concentração de material argilomineral (SIQUEIRA e APRILE, 2012). Embora a Ilha de Itacolomi esteja localizada longe da costa, a inexistência de barreiras semelhante a verifica na região amazônica pode favorecer o arraste de contaminantes até a região da ilha.

Embora as concentrações encontradas para os metais $\mathrm{Cd}$, As e Cu estejam dentro da faixa de segurança estabelecido pelo CONAMA 344/04, elas podem vir a servir como referencia para um acompanhamento da qualidade do sedimento.

Essas concentrações ficaram abaixo de valores encontrados em outros trabalhos que avaliaram regiões impactadas por atividades antrópicas.

Nas três regiões do Estuário de Targus, Portugal, os valores médios para o Cd de 4,1 $\mu \mathrm{g}$.g1 está acima do valor médio percebido no presente trabalho, porém os autores apontam para um decréscimo no lançamento de poluentes na região (FRANÇA et al., 2005). Na região Odiel e Cádiz, duas reservas na Espanha, os autores evidenciam na análise do sedimento uma média para o cádmio de 0,3 $\mu \mathrm{g}$.g-1 para Cádiz e de $12 \mu \mathrm{g}$.g-1 para Odiel, os autores apontam a média mais elevada a fatores antrópicos (USERO et al., 2003).

Os valores encontrados para o cobre seguiram a mesma tendência do cádmio ficando abaixo do nível 3. Na região da ilha das Bermudas, o valor apresentado para o cobre é extremamente elevado no sedimento, $1280 \mu \mathrm{g}$.g-1, quase 390 vezes maior que o valor apresentado nesta análise, porém os autores apontam para a região estar repleta de estaleiros como também para a presença de tinta antiincrustante das embarcações (TURNER, 2010).

Nas reservas de Cádiz e Odiel, apresentam-se dois resultados importantes para o cobre,

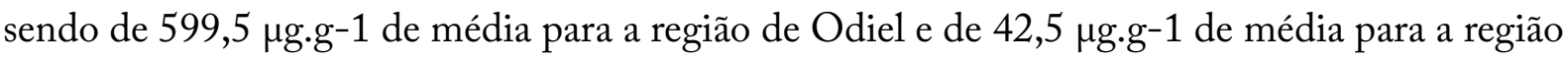
de Cádiz, justificam os autores a presença de ação antropogênica intensa na região de Cádiz (USERO et al., 2003), mesmo assim o valor apresentado para Odiel é extremamente mais elevado que o apresentado neste trabalho.

As concentrações de As estando abaixo o nível 3, indica uma baixa probabilidade de promoverem efeitos adversos à biota local. Porém, as concentrações encontradas no ouriço do mar (Tabela 3) e no A. Surinamensis (tabela 2) sugeri que possa haver uma relação.

Dentre os 4 metais o mercúrio foi o que apresentou valores entre o nível 3 e 4 . Mas esses valores estão abaixo do que já foi observado por outros autores que avaliaram regiões antropizadas. Nas regiões de Odiel e Cádiz, Espanha os valores para mercúrio variaram em média de 0,3 $\mu \mathrm{g} \cdot \mathrm{g}^{-1}$ para Cádiz e 0,7 $\mu \mathrm{g} \cdot \mathrm{g}^{-1}, 0,9 \mu \mathrm{g} \cdot \mathrm{g}^{-1}$ e à 1,6 $\mu \mathrm{g} \cdot \mathrm{g}^{-1}$ para Odiel, em sedimento, onde os autores sugerem área comprometida pela presença do mercúrio. No sedimento de fundo do Estuário de Santos, Siqueira et al (2005) encontraram vários pontos comprometidos pela presença de mercúrio. Siqueira et al (2005) atribuíram a emissão de esgoto pelo emissário submarino e também pelo despejo de mercúrio pelos rios que vem da Cidade de Cubatão. 
Não foi possível estabelecer nenhuma relação entre possíveis fontes e as concentrações dos metais encontradas no sedimento ao redor da Ilha de Itacolomi. A influência do tráfego de navios, embarcações pesqueiras e de lazer e a proximidade com os portos de Paranaguá e de São Francisco do Sul precisam ser melhor investigadas.

\section{Conclusão}

Pode-se concluir que até a data da coleta das espécies, os resultados apresentados após a análise são sugestivos de que a área pode encontrar-se livre da pressão de contaminação pelos metais mercúrio, arsênio, cádmio, chumbo e cobre. Porém, é necessário a execução de outros trabalhos na região da ilha de Itacolomi, para se observar se os valores determinados estão em ascensão ou se os valores são variações sazonais.

Um monitoramento sistemático também se faz necessário na área de estudo, a fim de elaborarmos um adequado plano de manejo para a região e sua sustentabilidade.

Os valores dos metais pesados analisados neste trabalho poderão contribuir para a elaboração de uma base de dados, como também, servir de referência para futuros trabalhos de biomonitoração na ilha de Itacolomi, Estado do Paraná, Brasil.

O presente estudo foi autorizado pelo SISBIO sob No 25698, 28/02/11.

\section{Referências}

AMADO-FILHO, G.M.A, SALGADO, L.T.A, REBELO,M.F.B, REZENDE, C.E.C, KAREZ, C.S.D, AND PFEIFFER, W.C.B. Heavy metals in benthic organisms from Todos os Santos Bay, Brazil. Brazilian Journal Biology, 68, n. 1, p. 95-100, 2008.

APHA. Standard methods for the examination of water and wastewater. 18th ed., American Public Health Association, Washington, DC, USA, 1998.

BRASIL. Portaria n. 685, de 27 de agosto de 1998. Princípios gerais para o estabelecimento de níveis máximos de contaminantes químicos em alimentos. Disponível em: < $\underline{\text { http:// }}$ www.anvisa.gov.br/legis/portarias/685 98.htm >. Acesso em: 26 de setembro de 2010.

BRASIL. Resolução no 344, de 25 de março de 2004. Diretrizes gerais e os procedimentos mínimos para a avaliação do material a ser dragado em águas jurisdicionais brasileiras. Disponível em: < http://www.novaambi.com.br/pdfs/344.pdf $>$ Acesso em: 15 de agosto de 2010 .

BONSIGNORE,M.;MANTA,D.S.; SHARIF,E.A.A-T.;DÁGOSTINO,F.;TRAINA, A.; QUINCI, E.M.; GIARAMITA, L.; MONASTERO, C.; BENOTHMAN, M.; SPROVIERI, M. Marine Pollution Bulletin, v. 128, p. 340-352, 2018.

BURGER, J.; GOCHFELD, M. Heavy metals in commercial fish in New Jersey. Environmental Research, v. 99, p. 403-412, 2005.

BUBACH, D.; CATÁN, S. P.; ARRIBÉRE, M.; GUEVARA, S. R. Bioindication of 
volatile elements emission by the Puyehue-Cordón Caulle (North Patagonia) volcanic event in 2011. Chemosphere, v. 88, p. 584-590, 2012

CETESB, Norma técnica L5 601. Ago 1994 / 4 paginas. <http://www.cetesb.sp.gov. br/normas/desativadas/L5.601 Sólidos\%20determinação\%20de\%20metais\%20por\%20 espectrofotometria\%20de\%20absorção\%20atômica\%20-\%20método\%20da\%20extr.pdf. $\geq$ Acessado em: 05 de junho de 2011.

CHI, Q.; ZHU, G.; LANGDON, A. Bioaccumulation of heavy metals in fishes from Taihu Lake, China. Journal of Environmental Sciences, v. 19, p. 1500-1504, 2007.

DALMAN, Ö.; DEMIRAK, A.; BALCI, A. Determination of heavy metals $(\mathrm{Cd}, \mathrm{Pb})$ and trace elements $(\mathrm{Cu}, \mathrm{Zn})$ in sediments and fish of the Southeastern Aegean Sea (Turkey) by atomic absorption spectrometry. Food Chemistry, v. 95, p. 157-162, 2006.

DAROS, F.A.L.M. Peixes recifais das Ilhas de Currais e Itacolomis, estado do Paraná. Pontal do Paraná, PR, 2010. Originalmente apresentada como dissertação de mestrado, Programa de Pós-Graduação em Sistemas Costeiros e Oceânicos, Centro de Estudos do Mar, Setor de Ciências da Terra, Universidade Federal do Paraná. Disponível em: $<\underline{\text { http:// }}$ hdl.handle.net/1884/26053> Acessado em: 29 de 08 de 2010.

FRANÇA, F.; VINAGRE, C.; CAÇADOR,I.; CABRAL, H. Heavy metal concentrations in sediment, benthic invertebrates and fish in three salt marsh areas subjected to different pollution loads in the Tagus Estuary (Portugal). Baseline/Marine Pollution Bulletin, v. 50, p. 993-1018, 2005.

GOULART,M.; CALLIXTO, M. Bioindicadores de qualidade de água como ferramenta em estudos de impacto ambiental. Revista da FAPAM, Faculdade de Pará de Minas, ano 2, n. 1, 2003.

GUSSO-CHOUERI, P.K.; ARAÚJO, G.S.; CRUZ, A.C.F.; STREMEL, T.R.O.; CAMPOS, S.X.; ABESSA, D.M.S.; RIBEIRO, C.A.O.; CHOUERI, R.B. Science of the Total environment, v. 628-629, p. 621-630, 2018.

MAANAN, M. Heavy metal concentrations in marine molluscs from the Moroccan coastal region. Environmental Pollution, v. 153, p. 176-183, 2008.

MACÍAS-ZAMORA, J.V.; RAMÍREZ-ALVAREZ, N. Tracing sewage pollution using linear alkylbenzenes (LABs) in surface sediments at the south end of the Southern California Bight. Environmental Pollution, v.130, p. 229-238, 2004.

MAHECHA, H.S.; Importância de ácidos graxos poliinsaturados presentes em peixes de cultivo e de ambiente natural para a nutrição humana. Boletim do Instituto de Pesca, v. 28, p. 101-110, 2002. 
MORGANO, M.A.; PEREZ, A.C.A.; MILANI, R.F.; MANTOVANI, D.M.B.; NEIVA, C.R.P.; FURLAN, E.F.; TOMITA, R.Y.; LOPES, R.G.; NETOS, M.J.L.; Mercúrio total em pescado da cadeia produtiva da Baixada Santista, estado de São Paulo, Brasil. Revista do Instituto Adolfo Lutz, v. 66, n. 2, p. 164-171, 2007.

NRIAGU, J.; BECKER, C. Volcanic emissions of mercury to the atmosphere: global and regional inventories. The Science of the Total Environment, v. 304, p. 3-12, 2003.

ONEM, S.A.; KUCUKSEZGIN, F.; KOCAK, F. Temporal and spatial biomonitoring of heavy metals in eastern Aegean coastal waters using Amphibalanus Amphitrite, Marine Pollution Bulletin, v. 62 p. 2548-2556, 2011.

OTIM, O.; JUMA, T.; SAVINELLI, R. The effect of a massive wastewater discharge on nearshore ocean chemistry. Environmental monitoring and assessment, v. 190, n. 4, p.180, 2018.

PORTAL OFICIAL DO MUNICÍPIO DE GUARATUBA Disponível em: $<\underline{\text { http:// }}$ www.guaratuba.pr.gov.br/site/index.php?option=com content\&task=view\&id=236\&Ite mid=181. $>$ acesso em: 10 de março de 2011

RADENAC, G.; MIRAMAND, D.F.; Bioaccumulation and toxicity of four dissolved metals in Paracentrotus lividus sea-urchin embryo. Marine Environmental Research, v. 51, n. 2, p. 151-166, 2001.

RUBIO, B.; NOMBELA, M.A.; VILAS, F.; Geochemistry of Major and Trace Elements in Sediments of the Ria de Vigo (NW Spain): an Assessment of Metal Pollution. Marine Pollution Bulletin, v. 40, n. 11, p. 968-980, 2000.

SIQUEIRA, G.W. AND APRILE, F.M. Distribuição de mercúrio total em sediment da Plataforma Continental Amazônica- Brasil. Acta Amazonica, v. 42, n. 2, 259-268, 2012.

SIQUEIRA, G.W.; BRAGA, E.S.; PEREIRA, S.F.P.; SILVA, E. Distribuição do mercúrio em sedimentos de fundo no Estuário de Santos - SP/ Brasil. Revista Escola de Minas, v. 58, n. 4, 309-316, 2005.

SOBIHAH, N.N.; ZAHARIN, A.A.; NIZAM, M.K.; JUEN, L.L.; KYOUNGWOONG, K. Bioaccumulation of heavy metals in maricultured fish, Lates calcarifer (Barramudi), Lutjanus campechanus (red snapper) and Lutjanus griséus (grey snapper). Chemosphere, v. 197, p. 318-324, 2018.

TÜRKMEN, A.; TÜRKMEN, M.; TEPE, Y.; AKYURT, I. Heavy metals in three commercially valuable fish species from Iskenderun Bay Northern East Mediterranean Sea, Turkey. Food Chemistry, v. 91, p. 167-172, 2005.

TURNER, A.; Marine pollution from antifouling paint particles. Marine Pollution 
Bulletin, v. 60, n. 2, p. 159-171, 2010.

ULUTURHAN, E.; KONTAS, A.; CAN, E. Sediment concentrations of heavy metals in the Homa Lagoon (Eastern Aegean Sea): Assessment of contamination and ecological risks. Marine Pollution Bulletin, v. 62, n. 9, p. 1989-1997, 2011.

USERO,J.; IZQUIERDO, C.; MORILLO, J.; GRACIA, I. Heavy metals in fish (Solea vulgaris, Anguilla anguilla and Liza aurata) from salt marshes on the southern Atlantic coast of Spain. Environment International, v. 29, p. 949-956, 2003.

VIEIRA, D.B e SHIBATTA, O.A. Peixes como indicadores da qualidade ambiental do ribeirão Espereança, município de Londrina, Paraná, Brasil. Biota Neotropical [online], v.7, n. 1, p. 0-0, 2007.

WANG,J.; LIU, R.H.; YU,P.; TANG,A.K.; XU,L.Q.:WANG J.Y.Study on the Pollution Characteristics of Heavy Metals in Seawater of Jinzhou Bay Procedia. Environmental Sciences, v. 13, p. $1507-1516,2012$.

ZHANG, W.; GUO, Z.; SONG, D.; DU, S.; ZHANG, L. Arsenic speciation in wild marine organisms and a health risk assessment in a subtropical bay of China. Science of the Total Environment, v. 626, p. 621-629, 2018. 